\title{
Robust inequality comparisons
}

\author{
Rolf Aaberge • Magne Mogstad
}

Received: 1 February 2010 / Accepted: 25 November 2010 / Published online: 31 December 2010

(C) The Author(s) 2010. This article is published with open access at Springerlink.com

\begin{abstract}
This paper is concerned with the problem of ranking Lorenz curves in situations where the Lorenz curves intersect and no unambiguous ranking can be attained without introducing weaker ranking criteria than first-degree Lorenz dominance. To deal with such situations, Aaberge (Soc Choice Welf 33:235-259, 2009) introduced two alternative sequences of nested dominance criteria for Lorenz curves, which proved to characterize two separate systems of nested subfamilies of inequality measures. This paper uses the obtained characterization results to arrange the members of two different generalized Gini families of inequality measures into subfamilies according to their relationship to Lorenz dominance of various degrees. Since the various criteria of higher degree Lorenz dominance provide convenient computational methods, these results can be used to identify the largest subfamily of the generalized Gini families, and thus the least restrictive social preferences, required to reach unambiguous ranking of a set of Lorenz curves. We further show that the weight-functions of the members of the generalized Gini families offer intuitive interpretations of higher degree Lorenz dominance, which generally has been viewed as difficult to interpret because they involve assumptions about third and higher derivatives. To demonstrate the usefulness of these methods for empirical applications, we examine the time trend in income and earnings inequality of Norwegian males during the period 1967-2005.
\end{abstract}

Keywords The Lorenz curve - Lorenz dominance - Rank-dependent measures of inequality The Gini coefficient • Generalized Gini families of inequality measures

JEL Classification D31 • D63

R. Aaberge $\cdot$ M. Mogstad ( $\bowtie)$

Research Department, Statistics Norway, P.O. Box 8131 Dep., N-0033 Oslo, Norway e-mail: magne.mogstad@gmail.com

R. Aaberge

e-mail: rolf.aaberge@ssb.no 


\section{Introduction}

Ranking Lorenz curves in accordance with first-degree Lorenz dominance means that the higher of two non-intersecting Lorenz curves is preferred. The normative significance of this criterion follows from the fact that the higher of two nonintersecting Lorenz curves can be obtained from the lower Lorenz curve by means of rank-preserving income transfers from richer to poorer individuals, which means that the criterion of first-degree Lorenz dominance is consistent with the Pigou-Dalton principle of transfers. When one Lorenz curve lies above another Lorenz curve, the higher Lorenz curve therefore displays less inequality than the lower Lorenz curve. However, since Lorenz curves may intersect, which is often the case in applied research, weaker ranking criteria than first-degree Lorenz dominance are required.

Although the theoretical literature offers more general dominance criteria for ranking intersecting Lorenz curves, ${ }^{1}$ these methods are generally viewed as hard to implement and the results difficult to interpret because they involve assumptions about third and higher derivatives (see e.g. [10,11]). Thus, most empirical studies rely exclusively on one or a few summary measures of inequality to achieve rankings of intersecting Lorenz curves. A concern is, however, that the conclusions reached are sensitive to the more or less arbitrary choice of inequality measures. It is, therefore, due time to start bridging the wide gap between the theoretical and the empirical strand of the literature concerned with rankings of intersecting Lorenz curves. That is the focus of this paper.

General Lorenz dominance criteria As demonstrated by Aaberge [5] two alternative dominance criteria emerge as attractive generalizations of first-degree Lorenz dominance; one that integrates the Lorenz curve from below (second-degree upward Lorenz dominance) and the other that integrates the Lorenz curve from above (second-degree downward Lorenz dominance). Since first-degree Lorenz dominance implies second-degree upward as well as downward Lorenz dominance, it follows that both methods preserve first-degree Lorenz dominance and thus are consistent with the Pigou-Dalton principle of transfers.

However, the transfer sensitivity of these criteria differs. While second-degree upward Lorenz dominance places more emphasis on transfers occurring in the lower part of the income distribution, second-degree downward Lorenz dominance is more sensitive to transfers that occur in the upper part of the income distribution. This means that the criterion of second-degree upward Lorenz dominance requires a transfer of money from a richer to a poorer person to be more equalizing the lower it occurs in the income distribution, provided that the proportion of individuals between the donors and receivers is fixed. By contrast, the criterion of second-degree downward Lorenz dominance requires this type of transfer to be more equalizing the higher it occurs in the income distribution.

For situations where neither upward nor downward Lorenz dominance of seconddegree provide unambiguous rankings of Lorenz curves, Aaberge [5] introduced two hierarchical sequences of Lorenz dominance criteria, and moreover, explored what

\footnotetext{
${ }^{1}$ See e.g. Kolm [21], Shorrocks and Foster [25], Davies and Hoy [15], Zoli [30] and Aaberge [1, 5].
} 
restrictions various Lorenz dominance criteria place on the weight-functions of the general Mehran-Yaari family of rank-dependent measures of inequality. In addition, by introducing general principles of transfer sensitivity, Aaberge [5] demonstrated that these criteria can be given a normative justification.

Inequality measures approach An alternative and more common strategy for achieving rankings of intersecting Lorenz curves is to apply the Gini coefficient. However, since no single measure can reflect all aspects of inequality exhibited by the Lorenz curve, the importance of using alternative measures to the Gini coefficient is universally acknowledged.

As proposed by Mehran [22], we may use weighed sums of the income share deviations as alternative rank-dependent measures of inequality to the Gini coefficient. The Mehran-Yaari family of rank-dependent measures of inequality, which includes the Gini coefficient, can alternatively be expressed as a weighted area between the Lorenz curve $(\mathrm{L}(\mathrm{u}))$ and its equality reference $(\mathrm{u})$. The chosen specification of the rank-dependent weight-function, which may be considered as the preference function of a social planner, clarifies whether concern about inequality is particularly related to the lower, the central or the upper part of the income distribution. Thus, the functional form of the weight-function exhibits the inequality aversion profile of the corresponding measure of inequality. Roughly speaking, we may say that a rank-dependent measure of inequality exhibits downside inequality aversion when the weight-function gives more emphasis to the deviation between the Lorenz curve and its equality reference, $\mathrm{u}-\mathrm{L}(\mathrm{u})$, for small $\mathrm{u}$ than for large $\mathrm{u}$. By contrast, when the weight-function gives more weight to the deviation $\mathrm{u}-\mathrm{L}(\mathrm{u})$ for large $\mathrm{u}$ than for small $\mathrm{u}$, we may say that the corresponding inequality measure exhibits upside inequality aversion.

Theoretical contributions The purpose of the theoretical part of this paper is to explore what restrictions various Lorenz dominance criteria place on the weightfunctions of two alternative generalized Gini families of rank-dependent measures of inequality, as well as to provide a normative justification of these criteria by relying on appropriate general principles of transfer sensitivity introduced by Aaberge [5]. As is demonstrated by Aaberge [5], second-degree Lorenz dominance forms a natural basis for the construction of two separate hierarchical sequences dominance criteria: one sequence places emphasis on changes that occur in the lower part of the Lorenz curve, whereas the other places emphasis on changes that occur in the upper part of the Lorenz curve. Both sequences of dominance criteria turn out to depart from the Gini coefficient. In particular, one requires higher degree of downside inequality aversion and the other higher degree of upside inequality aversion than what is exhibited by the Gini coefficient. This means that the Gini coefficient in general favors neither the lower nor the upper part of the Lorenz curves. In Section 2, we make two theoretical contributions. We first show that if we restrict the ranking problem to Lorenz curves with equal Gini coefficients, seconddegree upward and downward dominance coincide in the sense that a Lorenz curve $\mathrm{L}_{1}$ that second-degree upward dominates a Lorenz curve $\mathrm{L}_{2}$ is always second-degree downward dominated by $\mathrm{L}_{2}$. Secondly, we characterize the relationship between ordering conditions for the Gini coefficient and the general family of rank-dependent measures of inequality, in the case of singly intersecting Lorenz curves. 
As shown by Aaberge [5], the hierarchical and nested structure of the dominance criteria allows us to identify the lowest degree of dominance required to reach unambiguous rankings of Lorenz curves. In Section 3, we characterize the relationship between Lorenz dominance criteria of various degree and alternative rank-dependent measures of inequality. By doing so, we connect the dominance and the inequality measures approach to the ranking of intersecting Lorenz curves. In particular, we show how the members of two different generalized Gini families of inequality measures can be divided into subfamilies according to their relationship to Lorenz dominance of various degrees. These subfamilies of inequality measures offer different inequality aversion profiles: one exhibits successively higher degrees of downside inequality aversion, whereas the other exhibits successively higher degrees of upside inequality aversion. Since the criteria of Lorenz dominance provide convenient computational methods, these results can be used to identify the largest subfamily of the generalized Gini families, and thus the least restrictive social preferences, required to reach unambiguous ranking of any given set of Lorenz curves. And from the weight-functions of these inequality measures, we obtain intuitive interpretations of higher degree Lorenz dominance, which generally has been viewed as difficult to interpret.

Empirical application The evolution of earnings and income inequality in developed countries over the last few decades is one of the most extensively researched topics in economics. In a widely cited review of the literature, Gottschalk and Smeeding [18] conclude that earnings and income inequality increased in most OECD countries during the 1980s and early 1990s. Moreover, they argue that many countries with fairly low levels of inequality experienced some of the largest increases in inequality. These conclusions rest on numerous empirical studies relying exclusively on one or a few summary measures of inequality, like the Gini coefficient. A concern is, however, that the conclusions reached are sensitive to the more or less arbitrary choice of inequality measure.

Section 4 examines the time trend in income and earnings inequality of Norwegian males during the period 1967-2005, showing how the dominance approach and the inequality measures approach can be combined in a coherent way in empirical analysis. First, we identify the lowest degree of dominance, and hence the least restrictive social preferences, required to reach unambiguous rankings of the Lorenz curves over this period. Second, we pin down the largest subfamily of the generalized Gini families that is consistent with the actual ranking of these Lorenz curves. Finally, to ease the interpretation of the inequality aversion exhibited in the dominance results, we compute the weight-functions of these inequality measures.

\section{The relationship between Lorenz dominance and the Gini coefficient}

The Lorenz curve $L$ for a cumulative income distribution $F$ with mean $\mu$ is defined by

$$
L(u)=\frac{1}{\mu} \int_{0}^{u} F^{-1}(t) d t, 0 \leq u \leq 1,
$$


where $F^{-1}(t)=\inf \{x: F(x) \geq t\}$ is the left inverse of $F$. Thus, the Lorenz curve $L(u)$ shows the share of total income received by the poorest $100 u$ per cent of the population.

Under the restriction of equal mean incomes, the problem of ranking Lorenz curves formally corresponds to the problem of choosing between uncertain prospects. This relationship has been utilized by Atkinson [9] to characterize the criterion of non-intersecting Lorenz curves in the case of distributions with equal mean incomes. This was motivated by the fact that in cases of equal mean incomes the criterion of non-intersecting Lorenz curves is equivalent to second-degree stochastic dominance, ${ }^{2}$ which means that the criterion of non-intersecting Lorenz curves obeys the Pigou-Dalton principle of transfers. The Pigou-Dalton principle of transfers states that an income transfer from a richer to a poorer individual reduces income inequality, provided that their ranks in the income distribution are unchanged.

To perform inequality comparisons with Lorenz curves we can deal with distributions with equal means, or alternatively simply abandon the assumption of equal means and consider distributions of relative incomes. The latter approach normally forms the basis of empirical studies of income inequality.

The standard ranking criterion of non-intersecting Lorenz curves, called firstdegree Lorenz dominance, is based on the following definition, ${ }^{3}$

Definition 2.1 A Lorenz curve $L_{1}$ is said to first-degree dominate a Lorenz curve $L_{2}$ if

$$
L_{1}(u) \geq L_{2}(u) \text { for all } u \in[0,1]
$$

and the inequality holds strictly for some $u \in\langle 0,1\rangle$.

A social planner who prefers the dominating one of non-intersecting Lorenz curves favors transfers of incomes which reduce the differences between the income shares of the donor and the recipient, and is therefore said to be inequality averse.

In order to examine the relationship between various Lorenz dominance criteria and the measurement of inequality, we will rely on the family of rank-dependent measures of inequality, ${ }^{4}$ defined by

$$
J_{P}(L)=1-\int_{0}^{1} P^{\prime}(u) d L(u)=1-\frac{1}{\mu} \int_{0}^{1} P^{\prime}(u) F^{-1}(u) d u,
$$

where $L$ is the Lorenz curve of the income distribution $F$ with mean $\mu$, and the weight-function $P^{\prime}$ is the derivative of a continuous, differentiable and concave function $P$ defined on the unit interval where $P(0)=0$ and $P(1)=1$. To ensure

\footnotetext{
${ }^{2}$ For a proof, see Hardy et al. [19] or Atkinson [9].

${ }^{3}$ Note that most analyses of Lorenz dominance apply a definition that excludes the requirement of strict inequality for some $\mathrm{u}$.

${ }^{4}$ Mehran [22] introduced the $J_{P}$-family by relying on descriptive arguments. For alternative normative motivations of the $J_{P}$-family and various subfamilies of the $J_{P}$-family we refer to Donaldson and Weymark [16], Weymark [26], Yaari [28] and Aaberge [2].
} 
that $J_{P}$ has the unit interval as its range, the condition $P^{\prime}(1)=0$ is imposed on $P$. As demonstrated by Yaari $[27,28]$ and Aaberge [2], the $J_{P}$-family represents a preference relation defined either on the class of distribution functions $(F)$ or on the class of Lorenz curves $(L)$, where $P$ can be interpreted as a preference function of a social planner. The preference function $P$ assigns weights to the incomes of the individuals in accordance with their rank in the income distribution. Therefore, the functional form of $P$ reveals the attitude towards inequality of a social planner who employs $J_{P}$ to judge between Lorenz curves. The most well-known member of the $J_{P}$-family is the Gini coefficient, which is obtained by inserting for $P(u)=2 u-u^{2}$ in (2).

As demonstrated by Yaari [28], the $J_{P}$-family of inequality measures characterizes the condition of first-degree Lorenz dominance when the functional form of the preference function $P$ is strictly concave. This means that $J_{P}$ satisfies the PigouDalton principle of transfers for concave $P$-functions. To deal with situations where Lorenz curves intersect a weaker principle than first-degree Lorenz dominance is called for. To this end, researchers regularly employ second-degree upward Lorenz dominance, defined by

Definition 2.2 A Lorenz curve $L_{1}$ is said to second-degree upward dominate a Lorenz curve $L_{2}$ if

$$
\int_{0}^{u} L_{1}(t) d t \geq \int_{0}^{u} L_{2}(t) d t \text { for all } u \in[0,1]
$$

and the inequality holds strictly for some $u \in\langle 0,1\rangle$.

The term upward dominance refers to the fact that the Lorenz curves are integrated from below. ${ }^{5}$ The integrated Lorenz curve can be considered as a sum of weighted income shares, where the weights decrease linearly with increasing rank of the income receiver in the income distribution. Thus, a social planner who prefers the second-degree upward dominating of two intersecting Lorenz curves pays more attention to inequality in the lower than in the upper part of the income distribution. As proposed by Aaberge [5], an alternative ranking criterion to second-degree upward Lorenz dominance can be obtained by integrating the Lorenz curve from above.

Definition 2.3 A Lorenz curve $L_{1}$ is said to second-degree downward dominate a Lorenz curve $L_{2}$ if

$$
\int_{u}^{1}\left(1-L_{1}(t)\right) d t \leq \int_{u}^{1}\left(1-L_{2}(t)\right) d t \quad \text { for all } u \in[0,1]
$$

and the inequality holds strictly for some $u \in\langle 0,1\rangle$.

\footnotetext{
${ }^{5}$ Note that second-degree upward Lorenz dominance is equivalent to a normalized version of thirddegree inverse stochastic dominance introduced by Muliere and Scarsini [23].
} 
Note that both downward and upward Lorenz dominance of second degree preserve first-degree Lorenz dominance, since first-degree Lorenz dominance implies second-degree upward as well as second-degree downward Lorenz dominance. Consequently, both dominance criteria are consistent with the Pigou-Dalton principle of transfers. The choice between second-degree upward and downward Lorenz dominance clarifies whether or not equalizing transfers between poorer individuals should be considered more important than those between richer individuals.

As recognized by Muliere and Scarsini [23], there is no simple relationship between third-degree stochastic dominance and third-degree upward inverse stochastic dominance. Thus, since second-degree upward Lorenz dominance is equivalent to third-degree upward inverse stochastic dominance, a general characterization of second-degree Lorenz dominance (or third-degree inverse stochastic dominance) in terms of ordering conditions for the utilitarian measures introduced by Kolm [20] and Atkinson [9] cannot be obtained. As demonstrated by Aaberge [5], however, the family of rank-dependent measures of inequality, defined by (2), turns out to form a convenient basis for judging the normative significance of second-degree and higher degrees of Lorenz dominance.

To judge the normative significance of criteria for ranking intersecting Lorenz curves, more powerful principles than the Pigou-Dalton principle of transfers are needed. To this end, Kolm [21] introduced the principle of diminishing transfers, which for a fixed difference in income considers a transfer from a richer to a poorer person to be more equalizing the further down in the income distribution it takes place. ${ }^{6}$ As indicated by Shorrocks and Foster [25] and Muliere and Scarsini [23], the principle of diminishing transfers is, however, not consistent with second-degree upward Lorenz dominance. However, Mehran [22] introduced an alternative version of the principle of diminishing transfers by accounting for the difference in the proportion of individuals between donors and recipients of the income transfers, rather than for the difference in income. This principle, denoted first-degree downside positional transfer sensitivity (DPTS) by Aaberge [5], proves to characterize seconddegree upward Lorenz dominance. By contrast, a social planner who considers a given transfer of money from a richer to a poorer person to be more equalizing the higher it occurs in the income distribution, provided that the proportions of the population located between the receivers and the donors are equal, favors the principle of first-degree upside positional transfer sensitivity (UPTS). The UPTS characterizes second-degree downward Lorenz dominance. To ensure equivalence between second-degree downward Lorenz dominance and $J_{P}$-measures as decision criteria, Aaberge [5] proved that it is necessary to restrict the preference functions $P$ to be concave with negative third derivatives. ${ }^{7}$ By contrast, the condition of positive third derivative of $P$ yields second-degree upward dominance.

An inequality averse social planner who supports the criterion of second-degree upward Lorenz dominance will act in line with the principle of first-degree DPTS and assign more weight to changes that take place in the lower part of the Lorenz curve than to changes that occur in the upper part of the Lorenz curve. By contrast,

\footnotetext{
${ }^{6}$ For a formal definition, see Kolm [21].

${ }^{7}$ Aaberge [1] demonstrated that $J_{P}$ defined by (2) satisfies Kolm's principle of diminishing transfers under conditions that depend on the shape of the preference function $P$ as well as on the shape of the income distribution F.
} 
the criterion of second-degree downward Lorenz dominance emphasizes changes that occur in the upper part of the Lorenz curve. Thus, an inequality averse social planner who employs the criterion of second-degree downward Lorenz dominance acts in favor of the Pigou-Dalton principle of transfers and the principle of firstdegree UPTS.

Since the third derivative of the $P$-function that corresponds to the Gini coefficient is always equal to 0 , the Gini coefficient neither preserves second-degree upward Lorenz dominance nor second-degree downward Lorenz dominance, except for the case when the inequality in Definitions 2.3A and 2.3B holds strictly for $u=1$ and $u=0$, respectively. ${ }^{8}$ Thus, the suggestion of Muliere and Scarsini [23] that the Gini coefficient is coherent with second-degree upward Lorenz dominance requires a definition of second-degree Lorenz dominance that abandons the condition of strict inequality (for some $u \in\langle 0,1\rangle$ ). However, by assuming that the Lorenz curves cross only once the following results hold. ${ }^{9}$

Proposition 2.1A Assume that $L_{1}$ and $L_{2}$ are singly intersecting Lorenz curves and $L_{1}$ crosses $L_{2}$ initially from above, and let $G\left(L_{1}\right)$ and $G\left(L_{2}\right)$ be the two corresponding Gini coefficients. Then the following statements are equivalent,

(i) $J_{P}\left(L_{1}\right)<J_{P}\left(L_{2}\right)$ for all $P$ such that $P^{\prime}(u)>0, P^{\prime \prime}(u)<0$ and $P^{\prime \prime \prime}(u)>0$ for all $u \in(0,1)$

(ii) $G\left(L_{1}\right) \leq G\left(L_{2}\right)$.

(Proof in Appendix).

Proposition 2.1B Assume that $L_{1}$ and $L_{2}$ are singly intersecting Lorenz curves and $L_{2}$ crosses $L_{1}$ initially from above, and let $G\left(L_{1}\right)$ and $G\left(L_{2}\right)$ be the two corresponding Gini coefficients. Then the following statements are equivalent,

(i) $J_{P}\left(L_{1}\right)<J_{P}\left(L_{2}\right)$ for all $P$ such that $P^{\prime}(u)>0, P^{\prime \prime}(u)<0$ and $P^{\prime \prime \prime}(u)<0$ for all $u \in(0,1)$

(ii) $G\left(L_{1}\right) \leq G\left(L_{2}\right)$.

The proof of Proposition 2.1B can be achieved by following the line of reasoning used in the proof of Proposition 2.1A. Note that Proposition 2.1A can be considered as a dual version of the results of Shorrocks and Foster [25] and Dardanoni and Lambert [14], which clarify the relationship between third-degree (upward) stochastic dominance, ordering conditions for the coefficient of variation and transfer sensitive measures of inequality. Proposition 2.2 gives results for the case of equal Gini coefficients. In this case, second-degree upward and downward Lorenz dominance "coincide" in the sense that a Lorenz curve $L_{1}$ that second-degree upward dominates a Lorenz curve $L_{2}$ will always be second-degree downward dominated by $L_{2}$. Thus,

\footnotetext{
${ }^{8}$ Aaberge [1] gave an alternative interpretation of this property by demonstrating that the Gini coefficient attaches an equal weight to a given transfer irrespective of where it takes place in the income distribution, as long as the income transfer occurs between individuals with the same difference in ranks.

${ }^{9}$ Zoli [30] provided a result similar to Proposition 2.1A for singly intersecting generalized Lorenz curves under the condition of equal means.
} 
$L_{1}$ can be attained from $L_{2}$ by transfers that obey the principle of first-degree DPTS, whereas $L_{2}$ can be attained from $L_{1}$ by transfers that obey the principle of firstdegree UPTS.

Proposition 2.2 Let $L_{1}$ and $L_{2}$ be Lorenz curves with equal Gini coefficients. Then the following statements are equivalent,

(i) $L_{1}$ second-degree upward dominates $L_{2}$

(ii) $L_{2}$ second-degree downward dominates $L_{1}$

(iii) $J_{P}\left(L_{1}\right)<J_{P}\left(L_{2}\right)$ for all $P$ with $P^{\prime}(u)>0$ and $P^{\prime \prime}(u)<0$ being such that $J_{P}$ obeys the principle of first-degree DPTS.

(iv) $J_{P}\left(L_{2}\right)<J_{P}\left(L_{1}\right)$ for all $P$ with $P^{\prime}(u)>0$ and $P^{\prime \prime}(u)<0$ being such that $J_{P}$ obeys the principle of first-degree UPTS.

(Proof in Appendix).

\section{The relationship between general criteria of Lorenz dominance and generalized Gini families of inequality measures}

To deal with situations where second-degree (upward or downward) Lorenz dominance does not provide unambiguous ranking of Lorenz curves, Aaberge [5] introduced two hierarchical sequences of nested Lorenz dominance criteria that proved to divide the family of rank-dependent measures of inequality (2) into two corresponding hierarchical systems of nested subfamilies. In this section, we use these results to divide two generalized Gini families of rank-dependent measures of inequality into two corresponding hierarchical systems of nested subfamilies that offer different inequality aversion profiles: one exhibits successively higher degrees of downside inequality aversion (satisfies higher degrees of DPTS), whereas the other exhibits successively higher degrees of upside inequality aversion (satisfies higher degrees of UPTS). Note that the motivation for introducing the principles of DPTS (UPTS) was to successively strengthen the emphasis of transfers taking place in the lower (higher) part of the income distribution. As explained in Aaberge [5], the degree of aversion to downside inequality increases with the degree of upward Lorenz dominance. A similar relationship holds between downward Lorenz dominance and aversion to upside inequality aversion. The highest degree of downside inequality aversion is achieved when focus is exclusively on the situation of the worst-off income recipient. Thus, the $J_{P}$-measure that is obtained as the preference function approaches

$$
P_{d}(t)= \begin{cases}0, & t=0 \\ 1, & 0<t \leq 1,\end{cases}
$$

can be considered as the $J_{P}$-measure that exhibits the highest degree of downside inequality aversion. Inserting (3) in (2) yields

$$
J_{P_{d}}(L)=1-\frac{F^{-1}(0+)}{\mu} .
$$

Hence, the inequality measure $J_{P_{d}}$ corresponds to the Rawlsian maximin criterion. As $J_{P_{d}}$ is compatible with the limiting case of upward Lorenz dominance, the Rawlsian 
(relative) maximin criterion preserves all degrees of upward Lorenz dominance and rejects downward Lorenz dominance.

By contrast, the $J_{P}$-measure that is obtained as $P$ approaches ${ }^{10}$

$$
P_{u}(t)= \begin{cases}1, & 0 \leq t<1 \\ 0, & t=1\end{cases}
$$

exhibits the highest degree of upside inequality aversion. Inserting (5) in (2) yields

$$
J_{P_{u}}(L)=1+\frac{F^{-1}(1)}{\mu} .
$$

Thus, $J_{P_{u}}$, which we will denote the relative minimax criterion, is "dual" to the Rawlsian (relative) maximin criterion in the sense that it is compatible with the limiting case of downward Lorenz dominance. When the comparison of Lorenz curves is based on the relative minimax criterion, the preferred Lorenz curve is the one for which the largest relative income is smallest. The only transfers that decrease inequality are those from the richest unit to anyone else.

As will be demonstrated below, the extreme inequality aversion measure (4) is associated with the extended Gini family of inequality measures introduced by Donaldson and Weymark [16] and Yitzhaki [29]. This family is defined by

$G_{k}(L)=1-k(k+1) \int_{0}^{1}(1-u)^{k-1} L(u) d u=1-\frac{(k+1)}{\mu} \int_{0}^{\infty}(1-u)^{k} F^{-1}(u) d u, k \geq 1$.

In order to introduce an alternative "generalized" Gini family of inequality measures, Aaberge $[1,4]$ draws on the practice from the statistical literature as motivation for using the moments of the Lorenz curve as a basis for defining the Lorenz family of inequality measures ${ }^{11}$

$$
D_{k}(L)=1-(k+1) \int_{0}^{1} u^{k-1} L(u) d u=1-\frac{k+1}{\mu k} \int_{0}^{\infty}\left(1-u^{k}\right) F^{-1}(u) d u, k=1,2, \ldots,
$$

which is associated with the relative minimax criterion (6). Note that $D_{1}=G_{1}$ is equal to the Gini coefficient. As was demonstrated by Aaberge [4], the Lorenz family of inequality measures $\left\{D_{k}: k=1,2, \ldots\right\}$ uniquely determines the Lorenz curve. Thus, we can, without loss of information, restrict the examination of inequality in an income distribution $F$ to the family $\left\{D_{k}: k=1,2, \ldots\right\}$ of inequality measures.

As shown by the following expression

$$
G_{k}(L)=1+(k+1) \sum_{j=1}^{k}(-1)^{j}\left(\begin{array}{c}
k \\
j
\end{array}\right) \frac{j}{j+1}\left(1-D_{j}(L)\right), \quad k=1,2, \ldots
$$

\footnotetext{
${ }^{10}$ Note that the normalization condition $P^{\prime}(0)=1$ is ignored in this case.

${ }^{11}$ The Lorenz family of inequality measures proves to be a subclass of the "illfare-ranked single-series Ginis" discussed by Donaldson and Weymark [16] and Bossert [12].
} 
there is a one-to-one correspondence between the Lorenz family and the integer subfamily $\left\{G_{k}(L): k=1,2, \ldots\right\}$ of the extended Gini family of inequality measures. Moreover, the extended Gini subfamily $\left\{G_{k}(L): k=1,2, \ldots, r\right\}$ is uniquely determined by the corresponding Lorenz subfamily $\left\{D_{k}(L): k=1,2, \ldots, r\right\}$ for any integer $r$. Note that expressions (7) and (8) show that as $k$ increases, $G_{k}$ places more weight on changes in the lower part of the Lorenz curve, while $D_{k}$ places more weight on changes in the upper part of the Lorenz curve.

Based on Theorems 3.1A, 3.1B, 3.2A and 3.2B in Aaberge [5], we will now demonstrate how the various Lorenz dominance criteria can be applied to evaluate the ranking properties of the Lorenz and the extended Gini families of inequality measures. Note that $\left\{G_{k}: k>0\right\}$ is a subfamily of $J_{P}$ formed by the following family of $P$-functions,

$$
P_{1 k}(t)=1-(1-t)^{k+1}, k \geq 0 .
$$

Differentiating $P_{1 k}$ defined by (10), we find that

$$
P_{1 k}^{(j)}(t)=\left\{\begin{array}{cc}
(-1)^{j-1} \frac{(k+1) !}{(k-j+1) !}(1-t)^{k-j+1}, & j=1,2, \ldots, k+1 \\
0 \quad, \quad j=k+2, k+3, \ldots
\end{array}\right.
$$

As can be observed from (11), the weight function $P_{1 k}$ of the extended Gini family of inequality measures is a member of the family $P_{1 i}^{*}$ (and $P_{1 i}^{* *}$ ) in Aaberge [5] for $k=i$, $i+1, \ldots$. Thus, we get the following result from Theorem 3.2A of Aaberge [5], ${ }^{12}$

Proposition 3.1 Let $L_{1}$ and $L_{2}$ be members of $L$. Then

(i) $L_{1} i^{\text {th }}$-degree upward dominates $L_{2}$

implies

(ii) $G_{k}\left(L_{1}\right)<G_{k}\left(L_{2}\right)$ for $k=i, i+1, i+2, \ldots$

Equation (11) implies that $P_{1 k}^{\prime \prime}(t)<0$ for all $t \in\langle 0,1\rangle$ when $k>0$ and thus that the $G_{k}$-measures satisfy the Pigou-Dalton principle of transfers for $k>0$. Moreover, $P_{1 k}^{\prime \prime \prime}(t)>0$ for all $t \in\langle 0,1\rangle$ when $k>1$. Hence all $G_{k}$ for $k>1$ preserve seconddegree upward Lorenz dominance. Further, the derivatives of $P_{k}$ alternate in sign up to the $(k+1)^{t h}$ derivative and $P_{1 k}^{(j)}(1)=0$ for all $j \leq k$. Thus, it follows from Theorem 3.2A of Aaberge [5] that $G_{k}$ preserves upward Lorenz dominance of degree $k$ and obeys the principles of DPTS up to and including $(k-1)^{t h}$ degree. The highest degree of downside inequality averse behavior occurs as $k \rightarrow \infty$, which corresponds to the inequality averse behavior of the Rawlsian (relative) maximin criterion. Thus, $G_{k}$ satisfies all degrees of upward Lorenz dominance as $k \rightarrow \infty$. At the other extreme, as $k=0$, the preference function $P_{0}(t)=t$, which means that $J_{P_{0}}$ does not obey the Pigou-Dalton principle of transfers or any principle of DPTS. The stated properties of the $G_{k}$-measures are summarized in the following proposition,

${ }^{12}$ Muliere and Scarsini [23] gave an alternative proof of Proposition 3.1. 
Proposition 3.2 The extended Gini family of inequality measures defined by (7) has the following properties,

(i) $G_{k}$ preserves upward Lorenz dominance of degree $k$ and all degrees lower than $k$.

(ii) $G_{k}$ obeys the Pigou-Dalton principle of transfers for $k>0$, and the principles of DPTS up to and including $(k-1)^{\text {th }}$ degree for $k>1$.

(iii) The sequence $\left\{G_{k}\right\}$ approaches 0 as $k \rightarrow 0$.

(iv) The sequence $\left\{G_{k}\right\}$ approaches the Rawlsian relative maximin criterion as $k \rightarrow \infty$.

As demonstrated by Aaberge [1], the Lorenz family of inequality measures is a subfamily of $J_{P}$ formed by the following family of $P$-functions,

$$
P_{2 k}(t)=\frac{1}{k}\left((k+1) t-t^{k+1}\right), k=1,2, \ldots
$$

Differentiating $P_{2 k}$ defined by (12) yields

$$
P_{2 k}^{(j)}(t)= \begin{cases}\frac{k+1}{k}\left(1-t^{k}\right), & , j=1 \\ -(k+1)(k-1)(k-2) \ldots(k-j+2) t^{k-j+1} & , j=2,3, \ldots, k+1 \\ 0 & , j=k+2, k+3, \ldots\end{cases}
$$

By noting from (13) that the weight-function $P_{2 k}$ of the Lorenz family of inequality measures is a member of the family $P_{2 i}^{*}$ in Aaberge [5] for $k=i, i+1, \ldots$, we obtain the following result from Theorem 3.2B of Aaberge [5],

Proposition 3.3 Let $L_{1}$ and $L_{2}$ be members of $L$. Then

(i) $L_{1} i^{\text {th }}$-degree downward dominates $L_{2}$

implies

(ii) $D_{k}\left(L_{1}\right)<D_{k}\left(L_{2}\right)$ for $k=i, i+1, i+2, \ldots$.

and $\frac{F_{1}^{-1}(1)}{\mu_{1}}<\frac{F_{2}^{-1}(1)}{\mu_{2}}$.

The results of a similar evaluation of the Lorenz family of inequality measures as that carried out for the extended Gini family are summarized in the following proposition,

Proposition 3.4 The Lorenz family of inequality measures defined by (8) has the following properties,

(i) $D_{k}$ preserves downward Lorenz dominance of degree $k$ and all degrees lower than $k$.

(ii) $D_{k}$ obeys the Pigou-Dalton principle of transfers for $k>0$, and the principles of UPTS up to and including $(k-1)^{\text {th }}$ degree for $k>1$.

(iii) The sequence $\left\{D_{k}\right\}$ approaches 0 as $k \rightarrow \infty$.

(iv) The sequence $\left\{k D_{k}+1\right\}$ approaches the relative minimax criterion as $k \rightarrow \infty$. 
Proposition 3.1 shows that the various degrees of upward Lorenz dominance are preserved by sub-families of the extended Gini measures of inequality, which divide the integer subscript subclass of the extended Gini family into nested subfamilies. Thus, the hierarchical sequence of nested upward Lorenz dominance criteria offers a convenient computational method for identifying the largest subfamily of the extended Gini family of inequality measures that is consistent with the actual ranking of Lorenz curves. As demonstrated by Proposition 3.3, the various degrees of downward Lorenz dominance divide the Lorenz family of inequality measures into a similar sequence of nested subfamilies.

\section{Empirical application: income and earnings inequality in Norway, 1967-2005}

Below, we combine the dominance and inequality measures approach to examine the time trend in income and earnings inequality of Norwegian males during the period 1967-2005.

Data Our data are based on administrative registers from Statistics Norway covering the entire resident population in Norway between 1967 and 2004. The unique individual identifier allows merging information about individual characteristics, like age, with data on annual income and earnings taken from tax registers in each year. In the analysis, we employ two measures of income. First, we use a measure of annual pre-tax income including all taxable income after deductions. ${ }^{13}$ Secondly, we use a measure of earnings including all market income, from wages and self-employment. Individuals with missing observation on income or earnings are excluded. In each year 1967-2004, we include the entire population of males aged 20-65 who were alive and resident in that year. The reason for focusing on males is their role of breadwinner and primary wage-earners over most of this period.

It should be noted that we in the empirical analysis exclude individuals with incomes lower than the 1 percentile or higher than the 99 percentile, to minimize any crossing of Lorenz curves due to measurement error and noise at the tails of the distributions. Note also that our results do not suffer from sampling errors, since we have data on the entire population. However, when using sample surveys, it is necessary to employ relevant statistical tests for evaluating whether the estimated differences are statistical significant or not. To this end, we refer to the methods proposed by Aaberge [3, 4], Csörgö et al. [13] and Schechtman et al. [24].

Empirical results Figure 1 reports Gini coefficients for income and earnings, suggesting that Norway has experienced a large increase in inequality since the early 1980s, like most other OECD countries. ${ }^{14}$ Both income and earnings inequality decrease in the first period until the early 1980s, bottoming out at a level below .24. The trends then turn sharply upwards, approaching .32 in the early 1990 s. While

\footnotetext{
${ }^{13}$ Taxable income includes most cash benefits, but not in-kind benefits. The reason is that we do not have data on in-kind benefits for the period 1967-2005. See e.g. Aaberge et al. [7] for an analysis of the distributional impact of in-kind benefits in Norway.

${ }^{14}$ See Almås et al. [8] for a detailed discussion of the time trend in inequality in Norway, focusing especially on how changes in the age structure affects inequality.
} 
Fig. 1 Gini-coefficient in income and earnings, 1970-2005

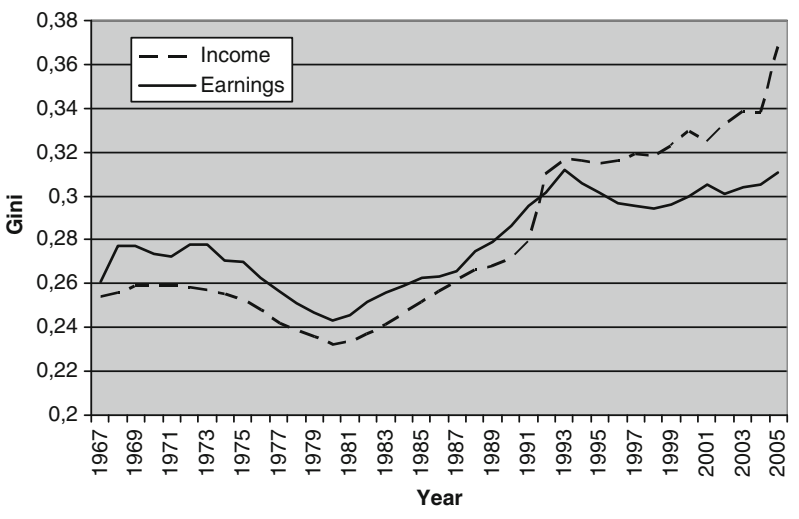

earnings inequality stabilizes over the last decade, income inequality increase further. A likely reason is the large increase in capital income in the upper part of the income distribution during this period [6]. It should be noted that the spike in inequality in the early 1990s was associated with a tax-reform, and that inequality is likely to have increased more steadily in the absence of this reform (see e.g. [17]). The same is true for the year 2005, reflecting changes in the tax reporting behavior due to the 2006 tax reform.

A concern with the above conclusions about the time-trend in inequality is that they might be sensitive to the choice of inequality measure. To investigate this, Aaberge [4] advocates using members of Gini's Nuclear Family, as these inequality measures have the same theoretical foundation, but supplement each other with regards to sensitivity in different parts of the distribution. For example, the Bonferroni coefficient is more sensitive to transfers in the lower part of the income distribution, compared to the Gini coefficients. When applying the Bonferroni coefficient, we find that it produces a qualitatively different time trend in income and earnings inequality. For example, the Bonferroni coefficient ranks 1970 as more equal than 1980 , in contrast to the ranking produced by the Gini coefficient. Instead of settling for such inconclusive evidence, we will in Tables 1 and 2 demonstrate how the methods proposed in this paper can be used to determine the least restrictive social preferences that are required to reach an unambiguous ranking of the two distributions.

In Table 1, we report dominance results for the Lorenz curves in income and earnings for the years 1970, 1980, 1990, and 2005. Panel 1 displays Lorenz dominance results for earnings, whereas Panel 2 reports Lorenz dominance results for income. In each panel, the results reported above (below) the diagonal refer to upward (downward) dominance. And for every dominance result, we first report the year that dominates and then the order of dominance. To ease the interpretation of the inequality aversion exhibited in the dominance results, Table 2 computes the weightfunctions of the two different generalized Gini families of inequality measures according to their relationship to Lorenz dominance of various degrees. Specifically, Table 2 reports the ratios of the weights of the median individual compared to the $1 \%$ poorest, the $5 \%$ poorest, the $30 \%$ poorest, the $30 \%$ richest, and the $5 \%$ richest. We immediately see that the extended Gini family of inequality measures, $G_{k}$ defined by (7), becomes increasingly sensitive to transfers that occur in the lower rather than the 


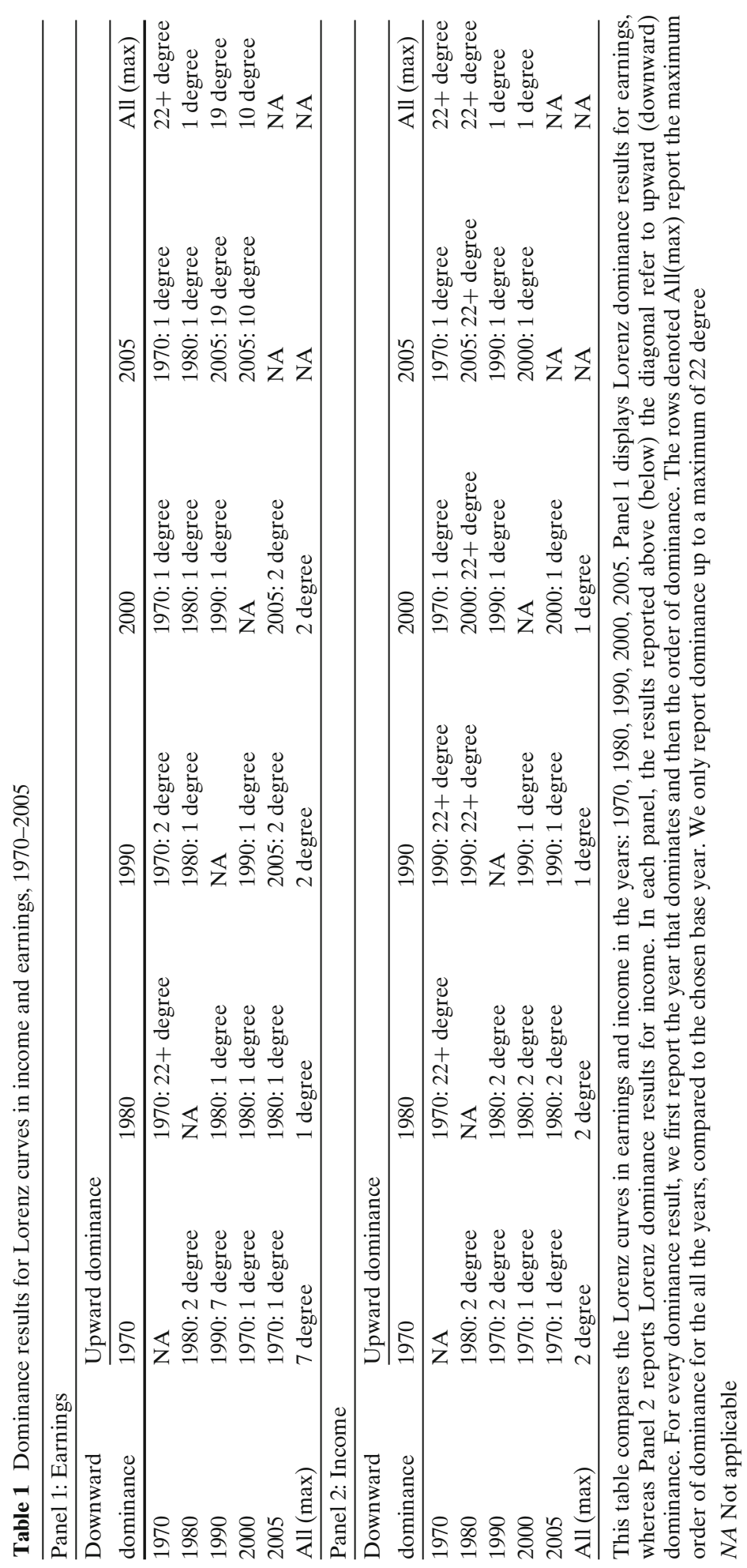


Table 2 Distributional weight of inequality measures by degree of upward and downward Lorenz dominance

\begin{tabular}{llllll}
\hline Upward Lorenz & \multicolumn{5}{l}{ Panel $\mathrm{A}: \mathrm{G}_{\mathrm{k}}$} \\
\cline { 2 - 6 } dom. of order $\mathrm{k}$ & $\mathrm{P}_{1 \mathrm{k}}^{\prime}(.01) /$ & $\mathrm{P}_{1 \mathrm{k}}^{\prime}(.05) /$ & $\mathrm{P}_{1 \mathrm{k}}^{\prime}(.3) /$ & $\mathrm{P}_{1 \mathrm{k}}^{\prime}(.7) /$ & $\mathrm{P}_{1 \mathrm{k}}^{\prime}(.95) /$ \\
& $\mathrm{P}_{1 \mathrm{k}}^{\prime}(.5)$ & $\mathrm{P}_{1 \mathrm{k}}^{\prime}(.5)$ & $\mathrm{P}_{1 \mathrm{k}}^{\prime}(.5)$ & $\mathrm{P}_{1 \mathrm{k}}^{\prime}(.5)$ & $\mathrm{P}_{1 \mathrm{k}}^{\prime}(.5)$ \\
\hline $\mathrm{k}=1$ & 1.98 & 1.90 & 1.40 & 0.60 & 0.10 \\
$\mathrm{k}=2$ & 3.92 & 3.61 & 1.96 & 0.36 & 0.01 \\
$\mathrm{k}=10$ & 926 & 613 & 29 & 0 & 0 \\
$\mathrm{k}=19$ & 433150 & 197842 & 598 & 0 & 0 \\
$\mathrm{k}=22$ & 3362282 & 1356998 & 1640 & 0 & 0 \\
\hline Downward Lorenz & Panel B: $\mathrm{D}_{\mathrm{k}}$ & & & & \\
\cline { 2 - 6 } dom. of order $\mathrm{k}$ & $\mathrm{P}_{2 \mathrm{k}}^{\prime}(.01) /$ & $\mathrm{P}_{2 \mathrm{k}}^{\prime}(.05) /$ & $\mathrm{P}_{2 \mathrm{k}}^{\prime}(.3) /$ & $\mathrm{P}_{2 \mathrm{k}}^{\prime}(.7) /$ & $\mathrm{P}_{2 \mathrm{k}}^{\prime}(.95) /$ \\
& $\mathrm{P}_{2 \mathrm{k}}^{\prime}(.5)$ & $\mathrm{P}_{2 \mathrm{k}}^{\prime}(.5)$ & $\mathrm{P}_{2 \mathrm{k}}^{\prime}(.5)$ & $\mathrm{P}_{2 \mathrm{k}}^{\prime}(.5)$ & $\mathrm{P}_{2 \mathrm{k}}^{\prime}(.5)$ \\
\hline $\mathrm{k}=1$ & 1.98 & 1.90 & 1.40 & 0.60 & 0.10 \\
$\mathrm{k}=2$ & 1.33 & 1.33 & 1.21 & 0.68 & 0.13 \\
$\mathrm{k}=7$ & 1.01 & 1.01 & 1.01 & 0.92 & 0.30 \\
$\mathrm{k}=22$ & 1.00 & 1.00 & 1.00 & 1.00 & 0.68 \\
\hline
\end{tabular}

Panel A considers upwards Lorenz dominance, whereas Panel B considers downward Lorenz dominance. In each panel, the first column states the degree of dominance, and columns $2-5$ compute the weight-functions of the generalized Gini families of inequality measures according to their relationship to Lorenz dominance of various degrees. Specifically, column 2 reports the ratio of the weights of the median individual compared to the $1 \%$ poorest, column 3 reports the ratio of the weights of the median individual compared to the 5\% poorest, column 4 reports the ratio of the weights of the median individual compared to the $30 \%$ poorest, column 5 reports the ratio of the weights of the median individual compared to the $30 \%$ richest, and column 6 reports the ratio of the weights of the median individual compared to the $5 \%$ richest. The weight-function $P_{1 k}^{\prime}(u)$ of $G_{k}$ is defined in Eq. 11 for $j=1$, while the weight function $P_{2 k}^{\prime}(\mathrm{u})$ of $D_{k}$ is defined in Eq. 13 for $j=1$

upper part of the distribution, as $k$ increases. It is also evident that the Lorenz family of inequality measures, $D_{k}$ defined by (8), becomes increasingly sensitive to transfers that occur in the upper rather than the lower part of the distribution, as $k$ increases. Hence, the choice between upward and downward dominance depends on the extent to which equalizing transfers between poorer individuals should be considered more important than those between richer individuals.

Consider first the inequality trend from 1970 and onwards. We see from Table 1 that first degree dominance is sufficient to rank 1970 as more equal than 2000 and 2005 , both for earnings and income. As stated above, first-degree dominance implies that all inequality measures that obey the Pigou-Dalton principle of transfer yield an unambiguous ranking of these Lorenz curves. However, when considering the years 1980 and 1990, more general dominance criteria are required.

Focusing attention first on upward dominance in earnings, we see that dominance of second degree is necessary to consistently rank 1970 as more equal than 1990, and further that dominance of (at least) 22 degree is required to unambiguously rank 1970 as more equal than 1980. From Proposition 3.1 we know that upward dominance of second degree implies that $G_{k}$ will consistently rank 1970 as more equal than 1990 for $k$ greater or equal to 2 . And further, that $G_{k}$ will consistently rank 1970 as more equal than 1980 for $k$ greater or equal 22. Table 2 shows that the weight-function associated with $G_{22}$ implies an extreme degree of downside inequality aversion, whereas $G_{2}$ is more sensitive than $G_{22}$ to income differences in the central and the upper part of the 
distribution. For instance, while $G_{2}$ assigns about twice as much weight to the $30 \%$ poorest compared to the median individual, $G_{22}$ weights the former individual 1640 times more than the latter individual. Hence, far more restrictive social preferences are required to unambiguously rank 1970 and 1980 compared to 1970 and 1990, according to upward dominance.

Turing attention to downward dominance in earnings, we see that the picture is reversed: 1970 is actually dominated by 1980 and 1990 of second and seventh degree, respectively. This illustrates that a consistent ranking of 1970, 1980, and 1990 depends crucially on the choice between upward and downward dominance criteria, that is, to what extent concern about inequality is particularly related to inequality in the lower, the central or the upper part of the distribution. From Proposition 3.3 we know that downward dominance of second degree implies that $D_{k}$ will consistently ranks 1980 as more equal than 1970 for $k$ greater or equal to 2, and 1990 as more equal than 1970 for $k$ greater or equal to 7 . From the weights-functions displayed in Table 2, we see that $D_{7}$ exhibits considerably more upside inequality aversion than $D_{2}$. Consequently, less restrictive social preferences are required to unambiguously rank 1970 and 1980 compared to 1970 and 1990 according to downward dominance.

Moving on to the inequality trend for the period 1980-2005, we see that 1980 first-degree dominates 1990, 2000, and 2005 for earnings, Hence, earnings inequality has unambiguously increased since the 1980. By contrast, downward dominance of second degree is necessary to consistently rank 1980 as more equal than 1990, 2000 and 2005 in terms of income. And in terms of upward dominance in earnings, 1980 is actually dominated of (at least) 22 degree by 1990, 2000 and 2005. It is also clear that 1990 first-degree dominates 2000, both for earnings and income. Yet for earnings, more restrictive social preferences are required to unambiguously rank 1990 and 2005. The same holds true when comparing 2000 and 2005: while 2000 first-degree dominates 2005 for income, downward dominance of second degree is necessary for 2000 to dominate 2005, and upward dominance of tenth degree is required for 2005 to dominate 2000 in terms of earnings.

\section{Conclusion}

Because Lorenz curves often intersect, other approaches than first-degree Lorenz dominance are called for. Although the theoretical literature offers more general dominance criteria for ranking Lorenz curves, these methods are generally viewed as hard to implement and the results difficult to interpret because they involve assumptions about third and higher derivatives (see e.g. [10,11]). Thus, most empirical studies rely exclusively on one or a few summary measures of inequality to achieve rankings of intersecting Lorenz curves. A concern is, however, that the conclusions reached are sensitive to the more or less arbitrary choice of inequality measures. The purpose of this paper has been to bridge the wide gap between the theoretical and the empirical strand of the literature concerned with rankings of intersecting Lorenz curves.

We first arrange the members of generalized Gini families of inequality measures into subfamilies according to their relationship to Lorenz dominance of various degrees. Since the various criteria of higher degree Lorenz dominance provide convenient computational methods, these results can be used to identify the largest 
subfamily of the generalized Gini families and thus the least restrictive social preferences required to reach unambiguous ranking of a set of Lorenz curves. From the weight-functions of these inequality measures we obtain intuitive interpretations of higher degree Lorenz dominance. To demonstrate the usefulness of these methods for empirical applications, we examine the time trend in income and earnings inequality of Norwegian males during the period 1967-2005.

Acknowledgements We thank two anonymous referees, Peter Lambert and the participants at the workshop "Inequality: New Directions" at Cornell University for useful comments and suggestions.

Open Access This article is distributed under the terms of the Creative Commons Attribution Noncommercial License which permits any noncommercial use, distribution, and reproduction in any medium, provided the original author(s) and source are credited.

\section{Appendix}

Proof of Proposition 2.1A. The statement (i) implies (ii) follows from Theorem 2.2A of Aaberge [5].

To prove the converse statement assume that (ii) holds and that $L_{1}$ and $L_{2}$ cross at $u=\mathrm{a}$. Then the following inequalities hold,

$$
\int_{0}^{a}\left(L_{1}(u)-L_{2}(u)\right) d u>0
$$

and

$$
\int_{0}^{1}\left(L_{1}(u)-L_{2}(u)\right) d u=\frac{1}{2}\left(G\left(L_{2}\right)-G\left(L_{1}\right)\right) \geq 0 .
$$

Since $L_{1}$ and $L_{2}$ cross only once (14) and (15) imply that

$$
\int_{0}^{u}\left(L_{1}(u)-L_{2}(u)\right) d u \geq 0 \text { for all } u \in[0,1]
$$

and the inequality holds strictly for some $u \in\langle 0,1\rangle$, and the desired result is obtained by applying Theorem 2.2A of Aaberge [5].

Proof of Proposition 2.2. The equivalence between (i) and (ii) follows by noting that

$$
\begin{gathered}
\int_{u}^{1}\left[\left(1-L_{1}(t)\right)-\left(1-L_{2}(t)\right)\right] d t=\int_{u}^{1}\left(L_{2}(t)-L_{1}(t)\right) d t= \\
\int_{0}^{1}\left(L_{2}(t)-L_{1}(t)\right) d t+\int_{0}^{u}\left(L_{1}(t)-L_{2}(t)\right) d t=\int_{0}^{u}\left(L_{1}(t)-L_{2}(t)\right) d t
\end{gathered}
$$

when $L_{1}$ and $L_{2}$ have equal Gini coefficients. 
The equivalence between (i) and (iii) and between (ii) and (iv) follows from Theorems 2.2A and 2.2B of Aaberge [5].

\section{References}

1. Aaberge, R.: Characterizations of Lorenz curves and income distributions. Soc. Choice Welf. 17, 639-653 (2000)

2. Aaberge, R.: Axiomatic characterization of the Gini coefficient and Lorenz curve orderings. J. Econ. Theory 101, 115-132 (2001)

3. Aaberge, R.: Asymptotic distribution theory of empirical rank-dependent measures of inequality. In: Nair, V. (ed.) Advances in Statistical Modeling and Inference-Essays in Honor of Kjell A. Doksum, World Scientific (2006)

4. Aaberge, R.: Gini's nuclear family. J. Econ. Inequal. 5, 305-322 (2007)

5. Aaberge, R.: Ranking intersecting Lorenz curves. Soc. Choice Welf. 33, 235-259 (2009)

6. Aaberge, R., Atkinson, A.B.: Top incomes in Norway. In: Atkinson, T., Piketty, T. (eds.) Top Incomes: A Global Perspective, pp. 448-482. Oxford University Press, Oxford (2010)

7. Aaberge, R., Bhuller M., Langørgen A., Mogstad M.: The distributional impact of public services when needs differ. J. Public Econ. 94, 549-562 (2010)

8. Almås, I., Havnes T., Mogstad M.: Baby booming inequality? Demographic change and inequality in Norway, 1967-2000. CESifo Working Paper 3200 (2010)

9. Atkinson, A.B.: On the measurement of inequality. J. Econ. Theory 2, 244-263 (1970)

10. Atkinson, A.B.: Multidimensional deprivation: contrasting social welfare and counting approaches. J. Econ. Inequal. 1, 51-65 (2003)

11. Atkinson, A.B.: More on the measurement of inequality. J. Econ. Inequal. 6, 277-283 (2008)

12. Bossert, W.: An approximation of the single-series Ginis. J. Econ. Theory 50, 82-92 (1990)

13. Csörgö, M., Gastwith, J.L., Zitikis, R.: Asymptotic confidence bands for the Lorenz and Bonferroni curves based on the empirical Lorenz curve. J. Stat. Plan. Inference 74, 65-91 (1998)

14. Dardanoni, V., Lambert, P.J.: Welfare rankings of income distributions: a role for the variance and some insights for tax reforms. Soc. Choice Welf. 5, 1-17 (1988)

15. Davies, J.B., Hoy, M.: Making inequality comparisons when Lorenz curves intersect. Am. Econ. Rev. 85, 980-986 (1995)

16. Donaldson, D., Weymark, J.A.: A single parameter generalization of the Gini indices of inequality. J. Econ. Theory 22, 67-86 (1980)

17. Fjærli, E., Aaberge, R.: Tax reforms, dividend policy and trends in income inequality empirical evidence based on Norwegian data. Statistics Norway, Discussion Paper, no. 284 (2000)

18. Gottschalk, P., Smeeding, T.: Cross-national comparisons of earnings and income inequality. J. Econ. Lit. 35, 633-687 (1997)

19. Hardy, G.H., Littlewood, J.E., Polya, G.: Inequalities. Cambridge University Press, Cambridge (1934)

20. Kolm, S.C.: The optimal production of social justice. In: Margolis, J., Guitton, H. (eds.) Public Economics. Macmillan, New York (1969)

21. Kolm, S.C.: Unequal inequalities II. J. Econ. Theory 13, 82-111 (1976)

22. Mehran, F.: Linear measures of inequality. Econometrica 44, 805-809 (1976)

23. Muliere, P., Scarsini, M.: A note on stochastic dominance and inequality measures. J. Econ. Theory 49, 314-323 (1989)

24. Schechtman, E., Shelef, A., Yitzhaki, S., Zitikis, R.: Testing hypotheses about absolute concentration curves and marginal conditional stochastic dominance. Econom. Theory 24, 1044-1062 (2008)

25. Shorrocks, A.F., Foster, J.E.: Transfer sensitive inequality measures. Rev. Econ. Stud. 14, 485497 (1987)

26. Weymark, J.: Generalized Gini inequality indices. Math. Soc. Sci. 1, 409-430 (1981)

27. Yaari, M.E.: The dual theory of choice under risk. Econometrica 55, 95-115 (1987)

28. Yaari, M.E.: A controversial proposal concerning inequality measurement. J. Econ. Theory 44, 381-397 (1988)

29. Yitzhaki, S.: On an extension of the Gini inequality index. Int. Econ. Rev. 24, 617-628 (1983)

30. Zoli, C.: Intersecting generalized Lorenz curves and the Gini index. Soc. Choice Welf. 16, 183196 (1999) 